\title{
Maternal high-fat feeding leads to alterations of brain glucose metabolism in the offspring: positron emission tomography study in a porcine model
}

\author{
Elena Sanguinetti $^{1,2} \cdot$ Tiziana Liistro $^{1} \cdot$ Marco Mainardi $^{3,4} \cdot$ Silvia Pardini $^{1}$.

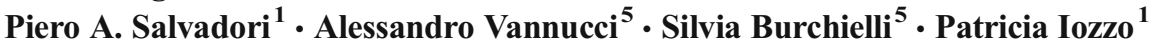

Received: 3 July 2015 / Accepted: 10 November 2015 /Published online: 5 January 2016

(C) Springer-Verlag Berlin Heidelberg 2016

\begin{abstract}
Aims/hypothesis Maternal obesity negatively affects fetal development. Abnormalities in brain glucose metabolism are predictive of metabolic-cognitive disorders.

Methods We studied the offspring (aged 0, 1, 6,12 months) of minipigs fed a normal vs high-fat diet (HFD), by positron emission tomography (PET) to measure brain glucose metabolism, and ex vivo assessments of brain insulin receptors $(\operatorname{IR} \beta)$ and GLUT4.

Results At birth, brain glucose metabolism and IR $\beta$ were twice as high in the offspring of HFD-fed than control mothers. During infancy and youth, brain glucose uptake, GLUT4 and IR $\beta$ increased in the offspring of control mothers and decreased in those of HFD-fed mothers, leading to a $40-85 \%$ difference $(p<0.05)$, and severe glycogen depletion, lasting until adulthood.

Conclusions/interpretation Maternal high-fat feeding leads to brain glucose overexposure during fetal development, followed by long-lasting depression in brain glucose metabolism in

Electronic supplementary material The online version of this article (doi:10.1007/s00125-015-3848-5) contains peer-reviewed but unedited supplementary material, which is available to authorised users.
\end{abstract}

Patricia Iozzo

patricia.iozzo@ifc.cnr.it

1 Institute of Clinical Physiology, National Research Council (CNR), via Moruzzi 1, Pisa 56124, Italy

2 Scuola Superiore di Studi Universitari Sant'Anna, Pisa, Italy

3 Institute of Neuroscience, National Research Council (CNR), Pisa, Italy

4 Institute of Human Physiology, Università Cattolica del Sacro Cuore, Rome, Italy

5 Fondazione Toscana Gabriele Monasterio (FTGM), Pisa, Italy minipigs. These features may predispose the offspring to develop metabolic-neurodegenerative diseases.

Keywords $\left[{ }^{18} \mathrm{~F}\right]$ FDG-PET $\cdot$ Brain $\cdot$ Fetal programming . Insulin sensitivity $\cdot$ Maternal high-fat feeding $\cdot$ Porcine model

\begin{tabular}{|c|c|}
\hline \multicolumn{2}{|c|}{ Abbreviations } \\
\hline$\left[{ }^{18} \mathrm{~F}\right] \mathrm{FDG}$ & 2-[ $\left[{ }^{18} \mathrm{~F}\right]$ fluoro-2-deoxyglucose \\
\hline $\mathrm{AU}$ & Arbitrary units \\
\hline FUR & Fractional $\left[{ }^{18} \mathrm{~F}\right] \mathrm{FDG}$ uptake rate \\
\hline GU & Glucose uptake \\
\hline HFD & High-fat diet \\
\hline i.m. & Intramuscular \\
\hline $\operatorname{IR} \beta$ & Insulin receptors \\
\hline ND & Normal diet \\
\hline PAS & Periodic acid-Schiff \\
\hline PET & Positron emission tomography \\
\hline ROI & Relative optical density \\
\hline RT & Room temperature \\
\hline
\end{tabular}

\section{Introduction}

Obesity represents one of the greatest current and future public health and societal challenges worldwide, leading to metabolic, cardiovascular and neurodegenerative complications [1-3]. Brain insulin resistance and cerebral glucose dysmetabolism are receiving great attention as common factors in the pathogenesis of obesity and cognitive impairment $[4,5]$. Insulin crosses the blood-brain barrier, and interacts with receptors localised in brain regions involved in the regulation of energy balance and glucose metabolism, but also in the modulation of learning and memory $[6,7]$. Furthermore, insulin-sensitive glucose transporters like GLUT4, and partially insulin- 
sensitive GLUT1, are expressed in different brain regions [7-9].

The epidemic of obesity involves an elevated and evergrowing number of pregnant women [10]. According to the fetal programming hypothesis, adaptations occurring in the developing offspring in response to the intra-uterine environment carry potentially permanent health consequences [11]. Indeed, maternal obesity is associated with increased fat mass at birth and altered fetal growth [12-14], and babies born to obese mothers are at an increased risk of being insulin resistant [15]. Animal and human studies have shown that maternal obesity during pregnancy predisposes the offspring to develop metabolic disorders [16-18] and increases the risk of cognitive decline later in life [19], associated with alterations in brain development, hypothalamic organisation and metabolism in the adult offspring [20, 21]. A reduction in cognitive development and performance was recently reported at early life stages or in pre-school/school children born to obese mothers $[22,23]$. Nevertheless, in vivo brain glucose metabolism, in the context of fetal programming and maternal obesity, has not been specifically investigated.

The aim of this study was to establish whether maternal high-fat feeding, started before gestation and continuing through lactation, affects the development and maturation of brain glucose metabolism in the offspring, from birth until adulthood. The study was carried out in minipigs, which are considered an affine model of human obesity and metabolism $[24,25]$. In vivo positron emission tomography (PET) with 2 - $\left[{ }^{18} \mathrm{~F}\right]$ fluoro-2-deoxyglucose $\left(\left[{ }^{18} \mathrm{~F}\right] \mathrm{FDG}\right)$ during hyperinsulinaemic-isoglycaemia and ex vivo measurements of brain-specific insulin receptors (IR $\beta$ ), insulin-dependent GLUT4 and glycogen content were used.

\section{Methods}

Animal model Adult female primiparous minipigs and their offspring (San Piero a Grado Farmhouse, Pisa, Italy), $n=10$ and $n=55$, respectively, were employed as described in Fig. 1. They were randomly assigned to 3 months of respective diets before conception. High-fat diet (HFD)-fed sows $(n=5)$ received a high-fat, high-energy diet $(+3,105 \mathrm{~kJ}$ from fat for 10 weeks, $+1,556 \mathrm{~kJ}$ from fat thereafter). A normal diet (ND) was supplied to lean animals $(n=5)$. Fresh water was provided ad libitum. Following the 3 month diet exposure, females were mated with the same male minipig and allowed to deliver spontaneously. They were maintained on respective diets throughout gestation and lactation. After weaning, offspring were fed with the standard diet.

$\left[{ }^{18} \mathrm{~F}\right]$ FDG-PET was employed to measure brain glucose metabolism in piglets aged 0, 1, 6 and 12 months, corresponding to birth, early infancy, youth and adulthood, respectively. Piglets born to one HFD and two ND mothers were studied at birth $(n=18)$ and then euthanised. Piglets born to the other four HFD and three ND mothers were studied at $1(n=37), 6$ $(n=24)$ and 12 months $(n=8)$.

Study session Anaesthesia was induced with tiletaminezolazepam $(10 \mathrm{mg} / \mathrm{kg}$ intramuscular [i.m.] Zoletil; Virbac Laboratories, Carros, France) and chlorpromazine $(1 \mathrm{mg} / \mathrm{kg}$, i.m. Largactil; Sanofi Aventis, Gentilly-Cedex, France), and maintained by an infusion of zolazepam $\left(2 \mathrm{mg} \mathrm{kg}^{-1} \mathrm{~h}^{-1}\right.$ i.m. Zoletil) in overnight-fasted animals. After body weight and basal glycaemia determination, catheters were placed into one ear vein for glucose, insulin and tracer administration, and into the contralateral ear vein for anaesthesia. At 6 and 12 months of age, blood samples were collected to measure fasting insulinaemia. Animals were positioned in the gantry of an ECAT HR+ tomograph (Siemens CTI, Knoxville, TN, USA), with a transaxial resolution of 5-8 $\mathrm{mm}$ full-width at half-maximum. A continuous infusion of insulin $\left(1 \mathrm{mU} \mathrm{min}{ }^{-1} \mathrm{~kg}^{-1}\right.$ ) was started $(t=0 \mathrm{~min})$. Isoglycaemia was maintained by a variable $20 \%$ glucose infusion (wt/vol.), adjusted according to glycaemia, as measured every $10 \mathrm{~min}$ by a glucometer. A transmission scan was first employed to correct subsequent emission data for photon attenuation. At $\sim t=45 \mathrm{~min},\left[{ }^{18} \mathrm{~F}\right] \mathrm{FDG}$ was injected and a $30 \mathrm{~min}$ dynamic acquisition was performed to assess time-activity levels in blood images of the aortic arch. Then, at $\sim t=75 \mathrm{~min}$, a static whole-body scan was performed to measure cerebral insulinmediated glucose disposal. After in vivo procedures, a subset of animals for each time point and group was euthanised for brain sample harvesting. The other subset was returned to the animal facility, monitored until complete recovery and restudied at the following time point. All the animals were generated and the experimental protocol was conducted in accordance with the D.L.116/92 implementation of European Economic Community directive 609/86 regarding the protection of animals used for experimental and other scientific purposes.

Image processing All sinograms were corrected for dead time, decay and photon attenuation and then reconstructed by standard algorithms in a $128 \times 128 \times 63$ matrix, with pixel size of $2.6 \times 2.6 \times 2.4$ and slice thickness of $2.4 \mathrm{~mm}$. Regions of interest were drawn on dynamic images corresponding to the aortic arch to extract the arterial tracer concentration over time (input function). The curve was integrated from 0 to the end of the scan to measure the AUC. Parametric images, representing the fractional uptake rate constant of $\left[{ }^{18} \mathrm{~F}\right] \mathrm{FDG}$ (FUR) in the brain $[26,27]$ were generated. FUR represents the inward $\left[{ }^{18} \mathrm{~F}\right] \mathrm{FDG}$ clearance $\left(\mathrm{ml} \mathrm{min}^{-1} \mathrm{ml}^{-1}\right)$ from blood to brain tissue, resulting from FDG transport and phosphorylation. It is computed as a ratio between the activity in brain tissue $(\mathrm{kBq} / \mathrm{ml}$ of brain), and the integral of the input function $([\mathrm{kBq} \times \mathrm{min}] / \mathrm{ml})$. 
Fig. 1 Offspring born to one HFD- and two ND-fed sows were studied at birth. Offspring born to remaining mothers were studied longitudinally. $\left[{ }^{18} \mathrm{~F}\right] \mathrm{FDG}$-PET was used to assess cerebral metabolic rates under hyperinsulinaemic-isoglycaemia. Subsets of offspring were euthanised at each time point, and cortical and hypothalamic samples collected to quantify GLUT4, IR $\beta$ and glycogen

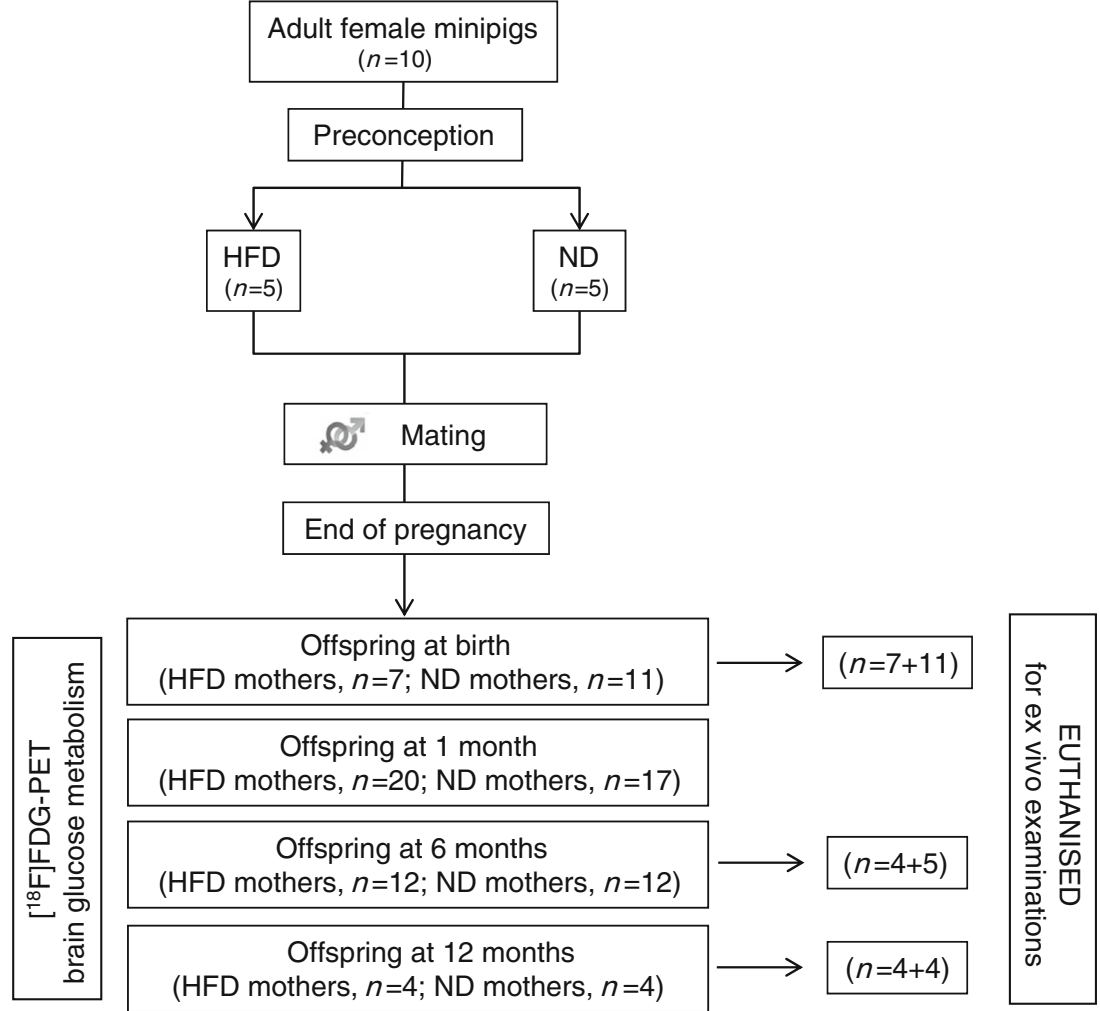

Regions of interest were manually drawn in the frontal, parietal, occipital, temporal cortices, cerebellum, brainstem and whole brain. Respective regional glucose uptake (GU) rates, expressed in $\mu \mathrm{mol} / \mathrm{min}$ per $100 \mathrm{ml}$ of brain tissue, were computed as a product of FUR and average glycaemia ( $\mu \mathrm{mol} / \mathrm{ml}$ ) during brain imaging, since brain $\mathrm{GU}$ is dependent on both cerebral extraction and glucose mass action [26, 27].

Western blot The expression of IR $\beta$ and GLUT4 in the frontal cortex and hypothalamus was investigated on $-80^{\circ} \mathrm{C}$ frozen biopsies collected upon euthanising. Proteins were extracted from homogenised samples with lysis buffer $(1 \%$ Triton $\mathrm{X}-100$ [wt/vol.], 10\% glycerol [vol./vol.], $20 \mathrm{mmol} / \mathrm{l} \mathrm{TrisHCl}$, $\mathrm{pH} 7.5,150 \mathrm{mmol} / \mathrm{l} \mathrm{NaCl}, 10 \mathrm{mmol} / \mathrm{l}$ EDTA, $0.1 \mathrm{mmol} / 1$ $\mathrm{Na}_{3} \mathrm{VO}_{4}, 10 \mu \mathrm{g} / \mathrm{ml}$ leupeptin, $10 \mu \mathrm{g} / \mathrm{ml}$ aprotinin and $1 \mathrm{mmol} / \mathrm{l} \mathrm{PMSF}$ ), and the total protein concentration in each sample was assessed with a Bradford assay kit (Bio-Rad, Hercules, CA, USA), using a BSA-based standard curve. Non-boiled protein extracts $(25 \mu \mathrm{g})$ were loaded on Tris- $\mathrm{HCl}$ $4-12 \%$ precast gels (Bio-Rad) and separated using SDS-PAGE $(1 \mathrm{~h}, 200 \mathrm{~V})$, then blotted on nitrocellulose membrane (BioRad) using a TurboBlot methanol-free system (Bio-Rad). Blots were blocked using the dedicated solution for the Odyssey imaging system (Li-Cor, Lincoln, NE, USA, 90 min, room temperature [RT]). The primary antibody solution was prepared in the same blocking solution diluted 1:1 in PBS-Tween $0.2 \%$ $(\mathrm{wt} / \mathrm{vol}$.) and contained either 1:1,000 anti-IR $\beta$ mouse monoclonal antibody (Millipore, Millerica, MA, USA) or anti-
GLUT4 rabbit polyclonal antibody (AbCam, Cambridge, UK). Incubation lasted overnight at $4^{\circ} \mathrm{C}$. Blots were then incubated with 1:20,000 secondary antibody conjugated to IR-Dye fluorophores (anti-rabbit IR-Dye-800 $\mathrm{nm}$ and anti-mouse IRDye-680 nm, Li-Cor, 1 h, RT) for visualisation on the Odyssey imaging platform. As an internal quantification standard, blots were probed with 1:20,000 anti- $\alpha$-tubulin mouse monoclonal antibody (Sigma-Aldrich, St Louis, MO, USA), and visualised with 1:20,000 anti-mouse IR-Dye- $800 \mathrm{~nm}$. The anti- $\alpha$-tubulin antibody was raised in mouse against sea urchin $\alpha$-tubulin. It recognises a C-terminal epitope [28], which is highly conserved in sea urchin, mouse and minipig (see electronic supplementary material [ESM] Methods for further information). The intensity of protein bands was quantified using the ImageStudio software (Li-Cor). The relative optical intensity (ROI) in each sample was computed, by dividing the IR $\beta$ or GLUT4 value by the corresponding $\alpha$-tubulin value.

Histological analysis Brain glycogen deposition was assessed by Periodic acid-Schiff (PAS) staining on $-80^{\circ} \mathrm{C}$ frozen biopsies collected upon euthanising. Cryosections ( $8 \mu \mathrm{m}$ thick), thawed for $30 \mathrm{~min}$ at RT, were fixed in $10 \%$ cold neutral buffered formalin (vol./vol.) (Bio-Optica, Milan, Italy) for 10 min and stained with PAS reaction (Bio-Optica) according to the manufacturer's protocol. Each section was documented at $\times 100$ and $\times 400$ magnification using a Axioskop optical microscope connected with an AxioCam MRc5 colourcamera and AxioVision analysis software (Carl Zeiss, 
Oberkochen, Germany). For each sample, three sections were randomly selected and interpreted using a semi-quantitative grading scale - established by an experimenter blinded to the conditions-according to the staining intensity (cortex, low $=1$ to high $=6$ arbitrary units $[\mathrm{AU}]$; hypothalamus, low $=1$ to high $=4 \mathrm{AU}$ ).

Statistical analysis All data are presented as means \pm SEM. Statistical analyses were performed using the IBM SPSS Statistics 21.0 software package (SPSS, Chicago, IL, USA). Group comparisons were performed by $t$ test for paired or independent samples as appropriate, and bivariate correlation was employed to evaluate the degree of association between variables of interest. Differences between groups were regarded as statistically significant when $p<0.05$.

\section{Results}

As expected, mothers exposed to HFD showed a $\sim 30 \%$ heavier body weight compared with ND mothers, both in pregravid and pregnant conditions $(p<0.05)$, despite no significant differences in glycaemia being detected (Fig. 2a, b). Instead, body weight was similar in the two groups of offspring at all time points (Fig. 2c), but glycaemia was significantly higher at birth in the offspring of HFD vs ND mothers. It declined during postnatal life in both groups, but the change was more pronounced in the offspring of HFD mothers, reaching lower glycaemic values during adulthood ( $p=0.01$ vs control offspring, Fig. 2d). A trend towards hyperinsulinaemia at 6 $(61.1 \pm 29.2 \mathrm{pmol} / 1$ vs $30.6 \pm 9.6 \mathrm{pmol} / \mathrm{l}, \mathrm{p}=\mathrm{NS})$ but no
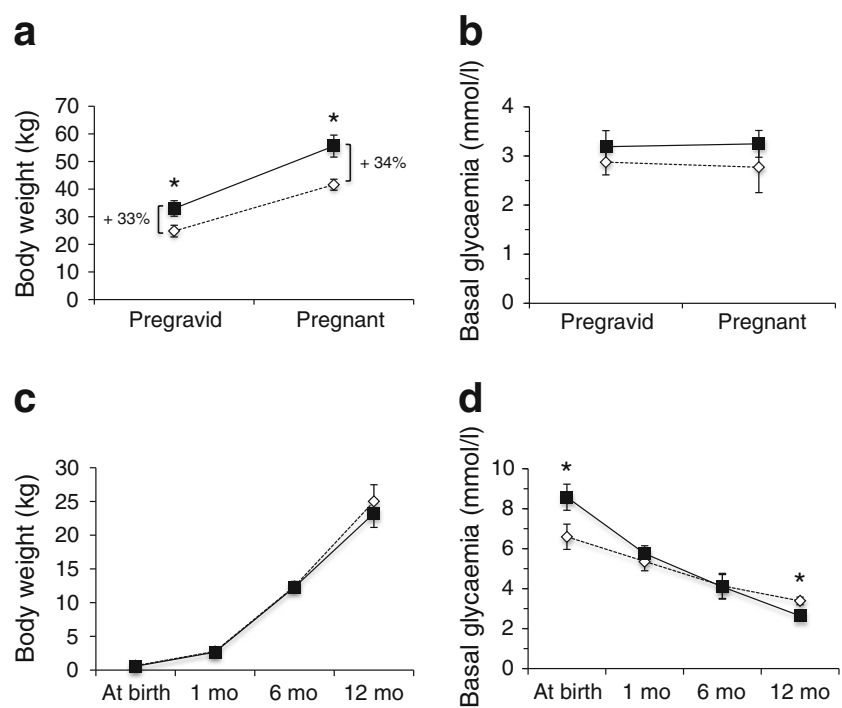

Fig. 2 Body weight and glycaemia were recorded before pregnancy and 2 weeks before delivery, in mothers (a, b), and at birth, 1, 6 and 12 months (mo) of age in the offspring (c, d). Black squares, offspring of HFD mothers; white diamonds, offspring of ND mothers. Values are means \pm SEM. ${ }^{*} p<0.05$. For sample sizes please see Fig. 1 differences at $12(21.5 \pm 13.9 \mathrm{pmol} / \mathrm{l}$ vs $19.1 \pm 6.3 \mathrm{pmol} / \mathrm{l}$, $\mathrm{p}=\mathrm{NS}$ ) months of age emerged in offspring of HFD mothers compared with controls.

\section{In vivo PET study}

$\left[{ }^{18} \mathrm{~F}\right]$ FDG-PET was employed to measure brain GU in the offspring at birth $(n=18), 1$ (infancy, $n=37), 6$ (youth, $n=24$ ) and 12 (adulthood, $n=8$ ) months of age, during hyperinsulinaemic-isoglycaemic stimulation. Images relative to one of the animals studied at birth were not available for the analysis (radiotracer injection out of the vein). Representative brain metabolic images are shown in Fig. 3a, b, and brain GU is given in Table 1 and Figs. 4a, 5a. In the offspring born to ND mothers, GU was lower at birth, increasing during the first month and plateauing thereafter. Conversely, the offspring of HFD mothers revealed a significantly higher GU at birth in most brain regions $(p<0.03)$, followed by a pronounced reduction at $1(p<0.002)$ and $6(p<0.05)$ months of age compared with the offspring of ND mothers, falling short of significance at 12 months of age.

\section{IR $\beta$ and GLUT4}

The expression of IR $\beta$ and GLUT4 in the frontal cortex and in the hypothalamus was measured in a subset of offspring at birth, 6 and 12 months of age, in biopsies collected upon

a
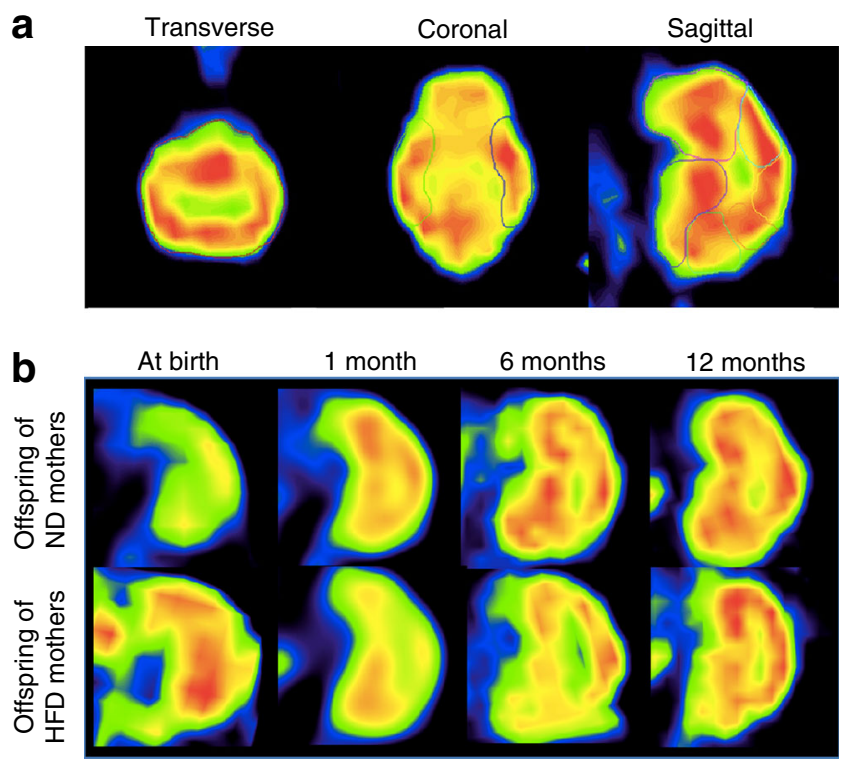

Fig. 3 Regions of interest were drawn in frontal, parietal, occipital, right/ left temporal cortices, cerebellum, brainstem and whole brain (a). Representative sagittal images document a higher GU at birth and deficiency thereafter in the offspring of HFD than ND mothers (b). For sample sizes please see Fig. 1 
Table 1 Brain GU rates in the offspring, from birth to adulthood

\begin{tabular}{|c|c|c|c|c|c|c|}
\hline Groups & Whole brain & Parietal cortex & Occipital cortex & Temporal cortex left & Temporal cortex right & Cerebellum \\
\hline \multicolumn{7}{|l|}{ Offspring at birth } \\
\hline HFD mothers $(n=7)$ & $17.8 \pm 3.8$ & $17.2 \pm 3.7$ & $16.2 \pm 3.8$ & $16.2 \pm 3.7$ & $16.4 \pm 3.6$ & $17.9 \pm 3.9$ \\
\hline ND mothers $(n=11)$ & $9.1 \pm 1.3$ & $9.2 \pm 1.4$ & $8.7 \pm 1.3$ & $8.7 \pm 1.2$ & $8.6 \pm 1.1$ & $9.8 \pm 1.4$ \\
\hline$p$ & 0.021 & 0.032 & 0.031 & 0.033 & 0.024 & 0.034 \\
\hline \multicolumn{7}{|l|}{ Offspring at 1 month } \\
\hline HFD mothers $(n=20)$ & $12.7 \pm 1.0$ & $13.1 \pm 1.0$ & $13.0 \pm 1.0$ & $12.8 \pm 1.0$ & $12.1 \pm 1.1$ & $13.5 \pm 1.1$ \\
\hline ND mothers $(n=17)$ & $19.5 \pm 1.5$ & $20.1 \pm 1.6$ & $19.7 \pm 1.5$ & $18.6 \pm 1.4$ & $18.3 \pm 1.4$ & $20.4 \pm 1.4$ \\
\hline$p$ & 0.001 & 0.001 & 0.001 & 0.002 & 0.001 & 0.001 \\
\hline \multicolumn{7}{|l|}{ Offspring at 6 months } \\
\hline HFD mothers $(n=12)$ & $11.7 \pm 1.1$ & $12.0 \pm 1.2$ & $12.0 \pm 1.1$ & $11.1 \pm 1.0$ & $11.1 \pm 1.0$ & $12.5 \pm 1.1$ \\
\hline ND mothers $(n=12)$ & $17.3 \pm 1.3$ & $17.8 \pm 1.5$ & $17.3 \pm 1.5$ & $15.5 \pm 1.5$ & $15.5 \pm 1.5$ & $17.8 \pm 1.3$ \\
\hline$p$ & 0.004 & 0.005 & 0.011 & 0.025 & 0.028 & 0.005 \\
\hline \multicolumn{7}{|l|}{ Offspring at 12 months } \\
\hline HFD mothers $(n=4)$ & $14.6 \pm 1.9$ & $14.9 \pm 1.9$ & $14.6 \pm 2.0$ & $14.0 \pm 1.8$ & $14.4 \pm 2.0$ & $15.8 \pm 2.0$ \\
\hline ND mothers $(n=4)$ & $17.0 \pm 2.9$ & $21.0 \pm 3.7$ & $20.6 \pm 3.2$ & $19.2 \pm 3.2$ & $19.1 \pm 3.4$ & $18.6 \pm 2.7$ \\
\hline$p$ & NS & NS & NS & NS & NS & NS \\
\hline
\end{tabular}

Data are means \pm SEM

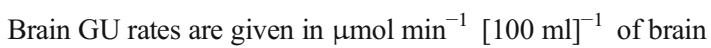

euthanising, and expressed as ROI vs corresponding $\alpha$ tubulin values. IR $\beta$ and GLUT4 values were consistent with insulin-mediated brain GU data. In the offspring of control mothers, their levels in the frontal cortex were low in newborns and higher in 6-month-old animals. Conversely, offspring of HFD mothers showed a fourfold higher IR $\beta$ expression in the frontal cortex at birth, and a significantly impaired expression of both IR $\beta$ and GLUT4 at 6 months ( $p<0.02$ vs control offspring). No significant difference between the two groups was detected at 12 months of age (Fig. 4b, c). The hypothalamic region showed similar trends, though group differences did not achieve full significance $(p=0.14$ and $p=0.09$, respectively) (Fig. 5b, c). IR $\beta$ and GLUT4 were reciprocally correlated within each region, and between neocortical and hypothalamic regions (Fig. 6a-d).

\section{Brain glycogen}

Brain glycogen content was assessed by PAS staining in frontal cortex and hypothalamic biopsies. Slides were interpreted using a semi-quantitative grading scale according to staining intensity (cortex, low $=1$ to high $=6 \mathrm{AU}$; hypothalamus, low $=1$ to high $=4 \mathrm{AU})$. A significant decrease in brain glycogen content in the frontal cortex was observed in 6-month-old offspring of HFD vs ND mothers $(p<0.01)$, persisting until 12 months of age $(p<0.05)$ (Fig. $4 \mathrm{~d}$, e). This pattern was not detected in the hypothalamus (data not shown).

\section{Discussion}

The high prevalence of obesity in young women of childbearing age may jeopardise the health of a growing number of affected mothers and their offspring. Its effect on developmental programming of adulthood diseases is a matter of intense investigation, since it provides opportunities for early prevention. Here, we focused on alterations of brain glucose metabolism, as recognised factors in the pathogenesis of obesity and cognitive decline.

The study was conducted in minipigs to combine longitudinal PET imaging and tissue sampling. PET is a wellrecognised method to quantify regional cerebral metabolism [29]. FDG is actively transported into brain cells and phosphorylated. FDG accumulation is assumed to be intracellular, since interstitial-to-cell transport is rapid, and FDG-6phosphate cannot enter glycolysis due to the lack of the 2'hydroxyl group. The bulk of brain GU is insulin independent, with a still debated insulin-induced effect [7]. Though we used the hyperinsulinaemic clamp to maximise the latter, our PET data cannot distinctively dissect these two components.

Several novel findings emerge from the present study. First, our study design offered the opportunity to delineate the physiological trajectory of brain glucose metabolism along the life course of the minipig offspring, showing that GU undergoes a very rapid rise, by doubling during the initial postnatal period, and stabilising thereafter. Old cross-sectional observations (1987) in neurologically diseased children ranging from 5 days to 15 years of age, undergoing $\left[{ }^{18} \mathrm{~F}\right]$ FDG-PET for diagnostic purposes, revealed a similar trend in the human brain, in 
a

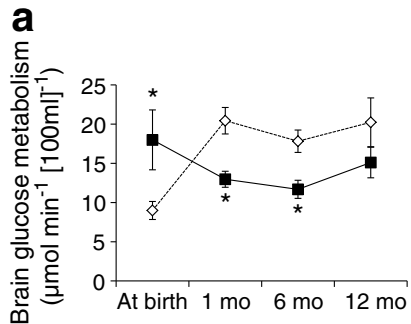

b
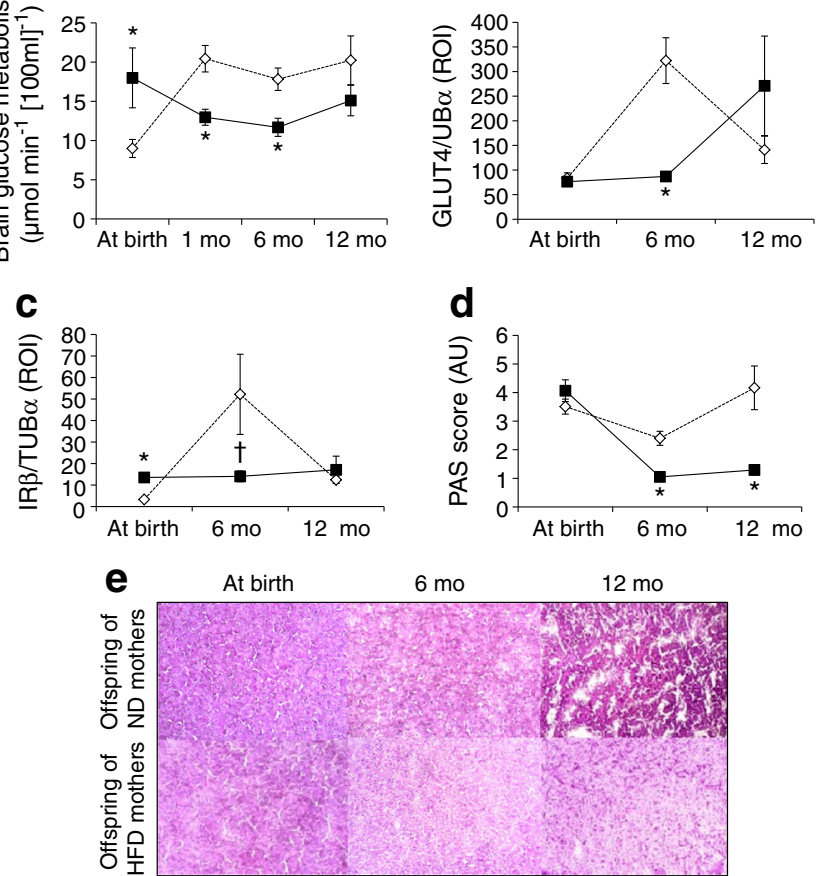

f

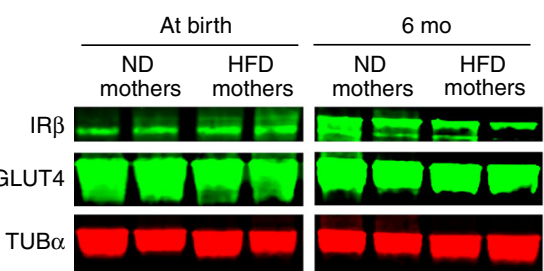

$12 \mathrm{mo}$
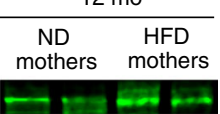

GLUT4

ig. 4 Cortical GU was calculated as a product of FUR and glycaemia during PET scanning (a). For the western blot, the ROI in each sample was computed by dividing the GLUT4 or IR $\beta$ level by the corresponding $\alpha$-tubulin (TUB $\alpha$ ) level $(\mathbf{b}, \mathbf{c})$; all samples were processed once in a single run to avoid the need of normalisation procedures. For glycogen quantification, PAS-stained sections were interpreted using a semi-quantitative grading scale, according to staining intensity (d), (low $=1$ to high $=6 \mathrm{AU})$. Representative sections in each group and time point are shown (e) $(\times 100$ magnification). Representative blots at each time point are shown (f). Black squares, offspring of HFD mothers; white diamonds, offspring of ND mothers. Values are means \pm SEM. ${ }^{*} p<0.05 ;{ }^{\dagger} p<0.02$ (Kruskal-Wallis test); mo, months. For sample sizes please see Fig. 1

which glucose metabolism was low at birth, doubling during infancy and progressively declining after the ninth year of age [30]. Confounders in the above human study included neurological disorders, cross-sectional comparisons and a limited life-period coverage. Our data were mostly longitudinal and referred to the entirely normal brain and to a more extended lifetime, from birth to adulthood.

The second and main finding of this study relates with the abnormalities in brain metabolism observed in the offspring undergoing maternal high-fat feeding during pregnancy and lactation. Our design was purposely meant to address longterm consequences of HFD in utero and during early brain development, and therefore the offspring were fed an ND during their life course. Our results revealed a remarkable increase a
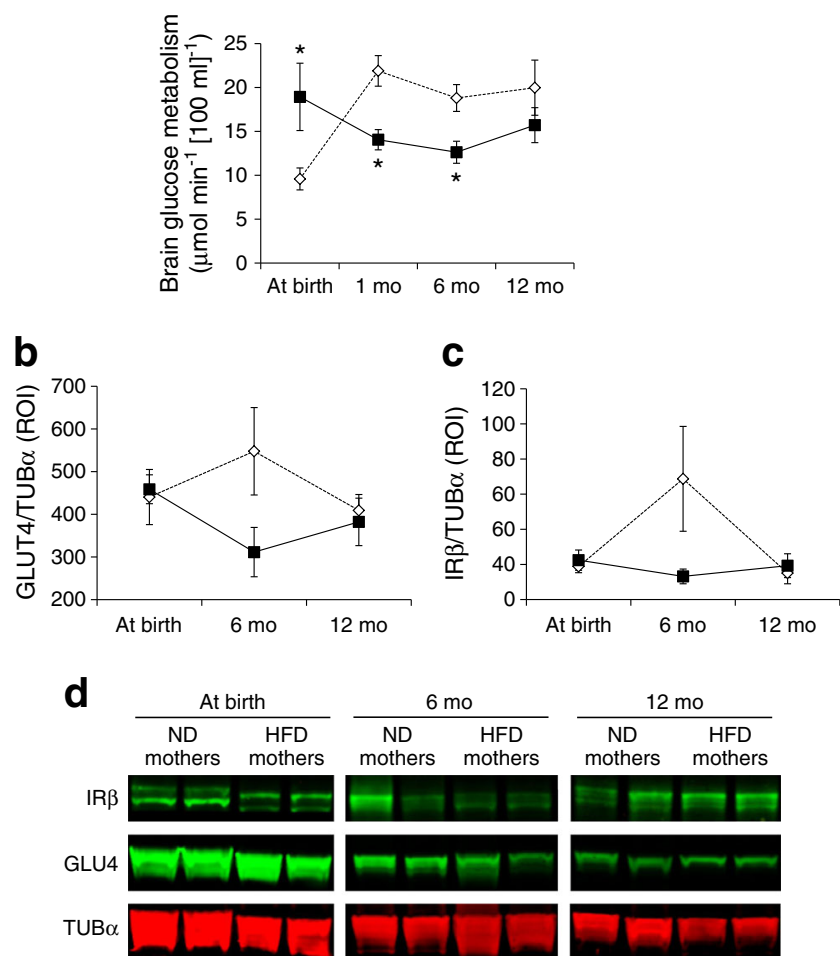

Fig. 5 Hypothalamic GU was calculated as the product of FUR and glycaemia during PET scanning (a). In the western blot, ROI in each sample was computed by dividing the GLUT4 or IR $\beta$ by the corresponding $\alpha$-tubulin (TUB $\alpha)$ level (b, c); the differences in GLUT4/ $\alpha$-tubulin $(p=0.09)(\mathbf{b})$ and IR $\beta / \alpha$-tubulin $(p=0.14)(\mathbf{c})$ levels at 6 months did not reach significance. Representative blots at each time point are shown (d); all samples were processed once in a single run to avoid the need of normalisation procedures. Black squares, offspring of HFD mothers; white diamonds, offspring of ND mothers. Values are means $\pm \mathrm{SEM}$. $*_{p}<0.05$. mo, months. For sample sizes please see Fig. 1
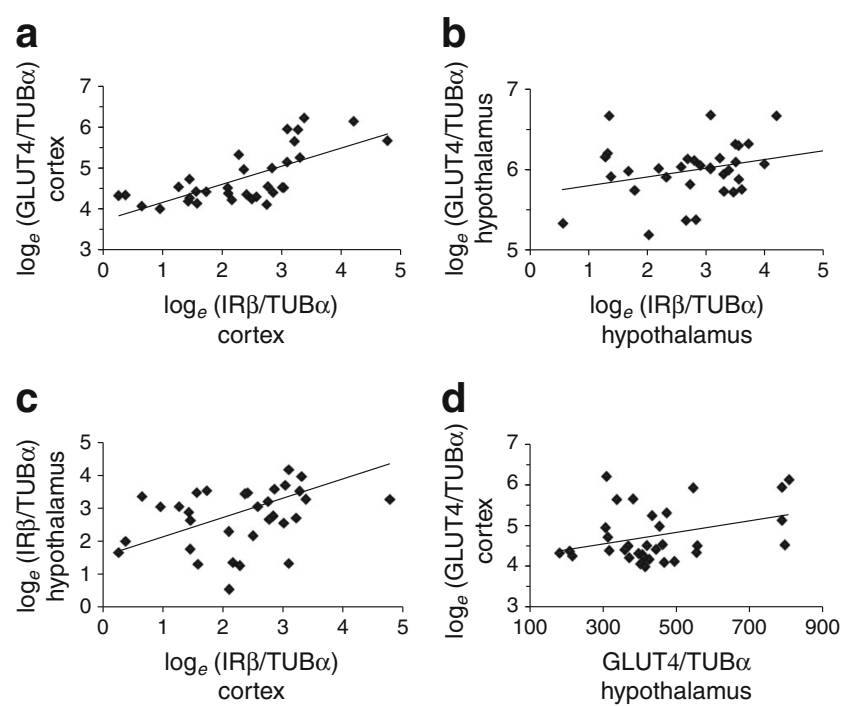

Fig. 6 A correlation was observed between IR $\beta$ and GLUT4 levels in the cortex (a) $(r=0.696, p<0.001)$ and hypothalamus (b) $(r=0.402$, $p=0.018)$. Cortical and hypothalamic IR $\beta$ and GLUT4 levels were associated (c) $(r=0.435, p=0.01)$; (d) $(r=0.346, p=0.045)$. TUB $\alpha, \alpha-$ tubulin. For sample sizes please see Fig. 1 
in brain glucose exposure in the offspring of HFD mothers at birth, involving homeostatic and cognitive-related regions. High levels of circulating glucose might explain this finding, since brain GU is the product of circulating glucose and brain glucose fractional extraction [31], and the latter was not altered in our study. Confirming previous data in rodents and humans, maternal HFD resulted in birth hyperglycaemia in the offspring, despite normal birthweight [32-35]. According to our data, the developing brain of minipigs responds to maternal HFD affecting the intra-uterine environment by the upregulation of IR $\beta$, glucose exposure and uptake. Third, our life course data addressed the interaction of high-energy exposure during prenatal development and normal lifestyle after birth. In these minipig offspring, a severe condition of brain glucose dysmetabolism ensued during lactation to last until adulthood. It was characterised by a $40-85 \%$ reduction in brain GU, IR $\beta$ and GLUT4 density, accompanied by a marked deficiency in glycogen deposition. At these time points, no significant difference in fasting glycaemia was noted, though a trend towards compensatory hyperinsulinaemia appeared in young adult offspring of HFD mothers. Therefore, different from birth, we hypothesise that changes in brain GU in these life phases might be attributed to alterations in the intrinsic brain fractional extraction capacity, and not to the effect of glycaemia, in accordance with GLUT4 results. Our observation agrees with recent results in 9-week-old rodents born to HFD dams, in which an acute stimulus failed to upregulate hypothalamic GU and neuropeptide-Y (NPY)/pro-opiomelanocortin (POMC) in vitro [36]. The downregulation of GU, IR $\beta$ and glycogen storage in our study may be interpreted either as a response to glucose toxicity, or as a maladaptive response to the high- to normal-energy diet switch. The former case is supported by the broad recognition that a protracted in vivo or in vitro exposure to hyperglycaemia negatively affects the glucose transport system and glycogen synthesis [37, 38]. The role of glucotoxicity in the brain $[31,39,40]$ and in early brain programming is less characterised, but might represent a plausible explanation to the development of cerebral hypometabolism as observed at 1 and 6 months. In fact, the protracted normalisation of glycaemia during growth seemed to alleviate its rate of progression, as brain metabolism and IR $\beta$ plateaued in adult offspring of HFD mothers. However, brain glycogen deposition was still markedly lower in adult animals born to HFD mothers.

Glucose is the unavoidable energy source of the brain. $\left[{ }^{18} \mathrm{~F}\right]$ FDG-PET studies have shown that its consumption grows rapidly in early life during brain maturation [30], and that brain glucose hypometabolism can anticipate, by many years, and predict the development of cognitive diseases [41-43]. Our study documents, in minipigs, that maternal HFD has a profound impact during the most sensitive period of brain development, leading to an early decrease as opposed to the physiological increase in glucose metabolism. Consequently, the slight deflection in GU, and the decline in
IR $\beta$ and GLUT4 observed during late adulthood in the control group due to physiological ageing, were detected (with equal or more severe magnitude) from very early life stages, and throughout life in the offspring of HFD mothers, accompanied by persistent and severe depletion of glycogen. Though astrocyte glycogen stores are small, they are important to sustain neuronal function, with key roles in memory formation and consolidation $[44,45]$. Altogether, we speculate that the above findings might indicate that the brain is metabolically aged and vulnerable towards neurodegeneration from infancy onwards in the offspring of HFD mothers. The density of IR $\beta$ and GLUT4 followed in vivo GU data and our age-related changes in GLUT4 are consistent with the available evidence in rodents $[46,47]$.

GU was similar in all brain areas in this study. Though regional differences in activation levels in response to insulin were detected by functional magnetic resonance imaging, reflecting blood oxygenation, a regional effect on GU is not clear. Our data in adult humans showed no significant regional differences in brain GU during a hyperinsulinaemic clamp [48]. We are not aware of studies on regional brain GU during early development in response to peripheral insulin stimulation.

Our study had some limitations. It was conducted in an animal model, and therefore its translational significance remains to be examined in humans. Anaesthesia during FDGPET may have led to a systematic underestimation of brain GU. However, it cannot be avoided during dynamic imaging, and the same protocol was always used, thereby minimising its influence on group comparisons. Our observation period spanned from birth to adulthood, but not elderly age. Animals studied at birth were born to dedicated sows, whereas the ones studied at 1, 6 and 12 months were evaluated longitudinally, with a subset undergoing euthanasia at each time point, and a subset continuing to the following time point. However, we have no reasons to expect a different evolution for animals studied at birth. Due to the demanding nature of the protocol, sample sizes were not numerous, though comparable with many published PET studies in animals and humans. The complementary use of ex vivo measurements of IR $\beta$, GLUT4 and glycogen reinforced the in vivo results. However, it is likely that the small sample sizes were responsible for the lack of significance in brain GU in later adulthood, and hypothalamic IR $\beta$ and GLUT4 levels at 6 months, which were affected by large variability. Although glycogen may undergo some degradation due to the time lag for tissue collection, the very small variability in results supports the reproducibility of procedures and errors. Maternal HFD was started before and continued through gestation and lactation. This design does not allow one to distinguish whether maternal obesity or the continuation of HFD during pregnancy elicited the observed abnormalities. However, it was chosen because fetal programming has been reported in conjunction 
with pre-gestational followed by gestational obesity in humans. Our primary purpose was to isolate early programming of the long-term health of the offspring, and therefore the offspring were raised on ND after lactation, and we did not address the interaction between early high-fat exposure and overfeeding during the life course.

In conclusion, cerebral glucose metabolism increases in the early postnatal period to plateau thereafter in the minipig offspring of lean mothers. The exposure to maternal HFD in the pre- and perinatal period results in brain glucose overexposure of homeostatic and cognitive regions at birth and brain glucose hypometabolism thereafter, associated with a reduced density of insulin receptors and insulin-dependent glucose transporters. Brain glycogen depletion is a long-lasting trait in adult minipig offspring born to HFD mothers. The study was conducted in animals and results may not be directly extrapolated to humans. It would be important to understand if preconception maternal weight loss and normalisation of dietary energy supply may safeguard the health of the offspring.

Acknowledgements The study was conducted at the Institute of Clinical Physiology of the CNR in Pisa and data have been presented at the European Congress of Obesity (ECO 2014, Oral Presentation, Abstract in Obesity Facts 2014;7(suppl 1):38, Sofia, Bulgaria).

Funding This study was funded by the EU (FP7) Project 'Developmental Origin of Healthy and Unhealthy Ageing: the Role of Maternal Obesity (DORIAN)' (grant no. 278603).

Duality of interest The authors declare that there is no duality of interest associated with this manuscript.

Contribution statement ES acquired, analysed and interpreted data, drafted and revised the manuscript; TL, MM, SP and AV provided substantial contribution to the acquisition of data. PAS contributed to the conception of the study design; SB was responsible for animal preparation and safety, and participated in the acquisition of data; PI designed the study, performed data analysis, wrote the manuscript, was the principal investigator and is the guarantor of the work. All the authors contributed to the critical revision of the manuscript and approved its final version for publication.

\section{References}

1. de la Monte SM, Wands JR (2008) Alzheimer's disease is type 3 diabetes-evidence reviewed. J Diabetes Sci Technol 2:1101-1113

2. Luchsinger JA (2008) Adiposity, hyperinsulinemia, diabetes and Alzheimer's disease. An epidemiological perspective. Eur J Pharmacol 585:119-129

3. Hallschmid M, Schultes B (2009) Central nervous insulin resistance: a promising target in the treatment of metabolic and cognitive disorders? Diabetologia 52:2264-2269

4. Moll L, Schubert M (2012) The role of insulin and insulin-like growth factor-1/FOXO-mediated transcription for the pathogenesis of obesity-associated dementia. Curr Gerontol Geriatr Res 384094
5. Craft S, Watson GS (2004) Insulin and neurodegenerative disease: shared and specific mechanisms. Lancet Neurol 3:169-178

6. Stockhorst U, de Fries D, Steingrueber HJ, Scherbaum WA (2004) Insulin and the CNS: effects on food intake, memory, and endocrine parameters and the role of intranasal insulin administration in humans. Physiol Behav 83:47-54

7. Bingham EM, Hopkins D, Smith D et al (2002) The role of insulin in human brain glucose metabolism: an 18fluoro-deoxyglucose positron emission tomography study. Diabetes 51:3384-3390

8. Ngarmukos C, Baur EL, Kumagai AK (2001) Co-localization of GLUT1 and GLUT4 in the blood-brain barrier of the rat ventromedial hypothalamus. Brain Res 900:1-8

9. Emmanuel Y, Cochlin LE, Tyler DJ, de Jager CA, Smith AD, Clarke K (2013) Human hippocampal energy metabolism is impaired during cognitive activity in a lipid infusion model of insulin resistance. Brain Behav 3:134-144

10. Kim SY, Dietz PM, England L, Morrow B, Callaghan WM (2007) Trends in pre-pregnancy obesity in nine states, 1993-2003. Obesity (Silver Spring) 15:986-993

11. Vickers MH (2011) Developmental programming of the metabolic syndrome - critical windows for intervention. World J Diabetes 2: $137-148$

12. O'Reilly JR, Reynolds RM (2013) The risk of maternal obesity to the long-term health of the offspring. Clin Endocrinol 78:9-16

13. Gaudet L, Tu X, Fell D, El-Chaar D, Wu SW, Walker M (2012) The effect of maternal class III obesity on neonatal outcomes: a retrospective matched cohort study. J Matern Fetal Neonatal Med 25: 2281-2286

14. Sewell MF, Huston-Presley L, Super DM, Catalano P (2006) Increased neonatal fat mass, not lean body mass, is associated with maternal obesity. Am J Obstet Gynecol 195:1100-1103

15. Catalano PM, Presley L, Minium J, Hauguel-de Mouzon S (2009) Fetuses of obese mothers develop insulin resistance in utero. Diabetes Care 32:1076-1080

16. Muhlhausler BS, Adam CL, Findlay CA, Duffield JA, McMillen IC (2006) Increased maternal nutrition alters development of the appetite-regulating network in the brain. FASEB J 20:1257-1259

17. Budge H, Stephenson T, Symonds ME (2007) Maternal nutrient restriction is not equivalent to maternal biological stress. Curr Drug Targets 8:888-893

18. Bouret SG (2009) Early life origins of obesity: role of hypothalamic programming. J Pediatr Gastroenterol Nutr 48(Suppl 1):S31-S38

19. Ross MG, Desai M, Khorram O, McKnight RA, Lane RH, Torday J (2007) Gestational programming of offspring obesity: a potential contributor to Alzheimer's disease. Curr Alzheimer Res 4:213-217

20. Plagemann A, Harder T, Janert U et al (1999) Malformations of hypothalamic nuclei in hyperinsulinemic offspring of rats with gestational diabetes. Dev Neurosci 21:58-67

21. Singh BS, Westfall TC, Devaskar SU (1997) Maternal diabetesinduced hyperglycemia and acute intracerebral hyperinsulinism suppress fetal brain neuropeptide $\mathrm{Y}$ concentrations. Endocrinology 138:963-969

22. Casas M, Chatzi L, Carsin AE et al (2013) Maternal pre-pregnancy overweight and obesity, and child neuropsychological development: two Southern European birth cohort studies. Int J Epidemiol 42:506-517

23. Basatemur E, Gardiner J, Williams C, Melhuish E, Barnes J, Sutcliffe A (2012) Maternal prepregnancy BMI and child cognition: a longitudinal cohort study. Pediatrics 131:56-63

24. Val-Laillet D, Layec S, Guérin S, Meurice P, Malbert CH (2011) Changes in brain activity after a diet-induced obesity. Obesity (Silver Spring) 19:749-756

25. Spurlock ME, Gabler NK (2008) The development of porcine models of obesity and the metabolic syndrome. J Nutr 138:397-402 
26. Ishizu K, Nishizawa S, Yonekura Y et al (1994) Effects of hyperglycemia on FDG uptake in human brain and glioma. J Nucl Med 35:1104-1109

27. Thie JA (1995) Clarification of a fractional uptake concept. J Nucl Med 36:711-712

28. LeDizet M, Piperno G (1987) Identification of an acetylation site of Chlamydomonas alpha-tubulin. Proc Natl Acad Sci U S A 84: 5720-5724

29. Varrone A, Asenbaum S, Vander Borght T, Booij J, Nobili F, Någren K (2009) EANM procedure guidelines for PET brain imaging using [18F]FDG, version 2. Eur J Nucl Med Mol Imaging 36: 2103-2110

30. Chugani HT, Phelps ME, Mazziotta JC (1987) Positron emission tomography study of human brain functional development. Ann Neurol 22:487-497

31. Liistro T, Guiducci L, Burchielli S et al (2010) Brain glucose overexposure and lack of acute metabolic flexibility in obesity and type 2 diabetes: a PET-[18F]FDG study in Zucker and ZDF rats. J Cereb Blood Flow Metab 30:895-899

32. Li M, Sloboda DM, Vickers MH (2011) Maternal obesity and developmental programming of metabolic disorders in offspring: evidence from animal models. Exp Diabetes Res 2011:592408

33. Barker DJ (2007) Obesity and early life. Obes Rev 8(Suppl 1):S45-S49

34. Desai M, Jellyman JK, Han G, Beall M, Lane RH, Ross MG (2014) Rat maternal obesity and high-fat diet program offspring metabolic syndrome. Am J Obstet Gynecol 211(3):237

35. Mingrone G, Manco M, Mora ME et al (2008) Influence of maternal obesity on insulin sensitivity and secretion in offspring. Diabetes Care 31:1872-1876

36. Chen H, Simar D, Morris MJ (2014) Maternal obesity impairs brain glucose metabolism and neural response to hyperglycemia in male rat offspring. J Neurochem 129:297-303

37. Kaiser N, Leibowitz G, Nesher R (2003) Glucotoxicity and betacell failure in type 2 diabetes mellitus. J Pediatr Endocrinol Metab $16: 5-22$
38. Rossetti L (2004) Glucose toxicity: effect of chronic hyperglycemia on insulin action. In: LeRoith D, Taylor SI, Olefsky JM (eds), Diabetes mellitus: a fundamental and clinical text, 3rd edn. Lippincott Williams \& Wilkins, Philadelphia, pp 939-951

39. Reaven GM, Thompson LW, Nahum D, Haskins E (1990) Relationship between hyperglycemia and cognitive function in older NIDDM patients. Diabetes Care 13:16-21

40. Patel MS, Srinivasan M (2010) Metabolic programming due to alterations in nutrition in the immediate postnatal period. J Nutr 140:658-661

41. Cunnane $\mathrm{S}$, Nugent $\mathrm{S}$, Roy $\mathrm{M}$ et al (2011) Brain fuel metabolism, aging, and Alzheimer's disease. Nutrition 27:3-20

42. de Leon MJ, Convit A, Wolf OT et al (2001) Prediction of cognitive decline in normal elderly subjects with 2-[(18)F]fluoro-2-deoxy-Dglucose/positron-emission tomography (FDG/PET). Proc Natl Acad Sci U S A 98:10966-10971

43. Mosconi L, de Santi S, Li J et al (2008) Hippocampal hypometabolism predicts cognitive decline from normal aging. Neurobiol Aging 29: 676-692

44. Bélanger M, Allaman I, Magistretti PJ (2011) Brain energy metabolism: focus on astrocyte-neuron metabolic cooperation. Cell Metab 14:724-738

45. Suzuki A, Stern SA, Bozdagi O et al (2011) Astrocyte-neuron lactate transport is required for long-term memory formation. Cell 144: $810-823$

46. Gómez O, Ballester-Lurbe B, Poch E, Mesonero JE, Terrado J (2010) Developmental regulation of glucose transporters GLUT3, GLUT4 and GLUT8 in the mouse cerebellar cortex. J Anat 217: 616-623

47. Sankar R, Thamotharan S, Shin D, Moley KH, Devaskar SU (2002) Insulin-responsive glucose transporters - GLUT8 and GLUT4 are expressed in the developing mammalian brain. Brain Res Mol Brain Res 107:157-165

48. Hirvonen J, Virtanen KA, Nummenmaa L et al (2011) Effects of insulin on brain glucose metabolism in impaired glucose tolerance. Diabetes 60:443-447 